\title{
Characterization of calcifications in human kidney by spectromicroscopy at the nanometer scale
}

Authors: Marta de Frutos (1), Alexandre Gloter (1), Dominique Bazin (2, 3), Marie-Christine Verpont $(4,5)$, Michel Daudon $(4,5,6)$, Emmanuel Letavernier $(4,5,6)$, Odile Stephan (1)

1. Laboratoire de Physique des Solides, CNRS, Université Paris Sud, Orsay, FRANCE

2. Laboratoire de Physique des Solides, CNRS - Université Paris Sud, Orsay, FRANCE

3. Laboratoire de Chimie de la Matière Condensée de Paris, UPMC, Collège de France, CNRS, Paris, FRANCE

4. Sorbonne Universités, UMR S 702, UPMC, Paris, FRANCE

5. UMR S 1155, INSERM, Paris, FRANCE

6. Physiology Unit, Hôpital Tenon, AP-HP, Paris, FRANCE

DOI: 10.1002/9783527808465.EMC2016.6705

Corresponding email: marta.de-frutos@u-psud.fr

Keywords: EELS, Principal components analysis, medical applications, kidney, calcium, phosphate

Randall's plaques are calcium phosphate deposits, at the origin of kidney stones. To date, little is known about the mechanisms involved in their formation. $\mu$ FTIR ( $\mu$ Fourier Transformed InfraRed spectroscopy) on samples from different kidneys indicate the presence of carbo-apatite, whitlockite and their co-existence with amorphous calcium phosphate phases. In the present study, our aim was to localize Randall's plaques at the early stages of their formation and to characterize their composition and crystallinity as a function of their localization, at the nanometer scale. Small pieces of papilla tip from healthy papillae of human kidneys were chemically fixed and embedded in epoxy resin. Ultrathin sections were analyzed by Transmission Electron Microscopy and Electron Energy-Loss Spectroscopy (EELS). Nano-calcifications were identified within vesicles, in many cases in close contact with collagen bundles (figure 1). These vesicles whose role and nature is to be determine could be the first step toward plaque formation. Selected Area Electron Diffraction (SAED) evidence that microvesicles contained a few nanocrystals whose diffraction pattern is compatible with the presence of crystalline apatite or whitlockite. Basic maps of light elements of biological interest ( $\mathrm{Ca}, \mathrm{P}, \mathrm{N}, \mathrm{O})$ confirm that dense deposits are mainly composed of $\mathrm{CaP}$ (figure 1). To go further, data were decomposed using the Hyperspy open source software for principal components analysis (PCA). The fine structure of the different EELS characteristic signals allows to investigate the composition of the nano-calcifications in order to try to discriminate between the various phases identified by $\mu$ FTIR (carbo-apatite, whitlockite, amorphous calcium phosphate). 



Figure 1: High angle Annular Dark Field (HAADF) images and EELS analysis revealed the presence of nanocalcifications made of CaP aggregated inside vesicles which "membranes" are nitrogen-rich and contain low amounts of $\mathrm{CaP}$ 